\title{
On binary constructions of quantum codes
}

\section{Gérard Cohen, Sylvia Encheva ${ }^{\dagger}$ Simon Litsyn ${ }^{\ddagger}$}

\begin{abstract}
We improve estimates on the parameters of quantum codes obtained by Steane's construction from binary codes. This yields several new families of quantum codes.
\end{abstract}

\section{Introduction}

Although quaternary constructions [3] yield good quantum codes, building quantum codes from binary ones results in efficient and easier to implement families. Such an approach was suggested by Calderbank and Shor [4] and Steane [8, 9]. Recently, Steane [10] proposed an enlargement of the Claderbank-Shor-Steane construction, leading to several families of codes with fixed minimum distance and growing length. In this paper, we further improve the estimates of codes parameters obtained from Steane's construction, present examples of new codes, and analyze asymptotical nonconstructive bounds.

An (additive stabilizer) quantum code of length $n$ with $2^{k}$ codewords and minimum distance $d$, is an eigenspace of a commutative subgroup of the group $E$ of tensor products of Pauli matrices. The commutativity condition is

$$
H_{x} \cdot H_{z}^{T}+H_{z} \cdot H_{x}^{T}=\mathbf{0},
$$

*Ecole Nationale Supérieure des Télécommunications, 46 rue Barrault, 75634 Paris, France; e-mail: cohen@inf.enst.fr

†Stord/Haugesund College Skaareg. 103, 5500 Haugesund, Norway; e-mail: sbe@hsh.no

$\ddagger$ DIMACS, on sabbatical leave from Department of Electrical Engineering-Systems, Tel Aviv University, 69978 Ramat Aviv, Israel; e-mail: litsyn@eng.tau.ac.il 
where $H_{x}$ and $H_{z}$ are $(n-k) \times n$ binary matrices which together form the stabilizer $\mathcal{H}=\left(H_{x} \mid H_{z}\right)$. The $2 n$-vectors $\left(u_{x} \mid u_{z}\right)$ satisfying $H_{x} \cdot u_{z}+H_{z} \cdot u_{x}=0$ form the code $\mathcal{C}$. These vectors are generated by $\mathcal{G}=\left(G_{x} \mid G_{z}\right)$, where

$$
H_{x} \cdot G_{z}^{T}+H_{z} \cdot G_{x}^{T}=\mathbf{0} .
$$

Define the generalized weight of a vector $\left(u_{x} \mid u_{z}\right)$ as the Hamming weight of the bitwise OR of $u_{x}$ and $u_{z}$. Let $\mathcal{C}^{\perp}$ be the dual of $\mathcal{C}$ with respect to the inner product $\left(\left(u_{x} \mid u_{z}\right),\left(v_{x} \mid v_{z}\right)\right)=u_{x} \cdot v_{z}+u_{z} \cdot v_{x}$. The minimum (quantum) distance $d$ of the quantum code $\mathcal{C}$ is the largest generalized weight of a vector in $\mathcal{C} \backslash \mathcal{C}^{\perp}$. This code has parameters $[[n, K, d]]$ where $K$ is $n-\log _{2}|\mathcal{C}|$.

Let $C[n, k, d](C[n, k]$ if $d$ is irrelevant) denote a binary linear code of length $n$, dimension $k$ and minimum distance $d$. For a survey of the theory of codes see e.g. [7].

The following construction was proposed by Steane [10].

Theorem 1 10] Let $C[n, k, d], C^{\perp} \subseteq C$, be a classical binary linear error correcting code with generator matrix $G$. Let $C$ be a subcode of a code $C^{\prime}\left[n, k^{\prime}>k+1, d^{\prime}\right]$ with generator matrix $\left(\begin{array}{c}G \\ G^{\prime}\end{array}\right)$. Then

$$
\mathcal{G}=\left(\begin{array}{cc}
G & 0 \\
0 & G \\
G^{\prime} & P G^{\prime}
\end{array}\right)
$$

where $P$ is a fix-point free map (for instance a permutation), generates a quantum code of parameters $\left[\left[n, k+k^{\prime}-n, \geq \min \left(d,\left[\frac{3 d^{\prime}}{2}\right\rceil\right)\right]\right]$.

Proof We sketch the proof for self-completeness. Consider any non-zero combination $\mathbf{u}=\left(u_{x} \mid u_{z}\right)$ of rows of $\mathcal{G}$. If no rows of the submatrix $\left(G^{\prime} \mid P G^{\prime}\right)$ are involved in the generation of $\mathbf{u}$, then the generalized weight of $\mathbf{u}$ is $\geq d$. Otherwise, since $\left(\begin{array}{c}G \\ P G^{\prime}\end{array}\right)$ also generates $C^{\prime}, u_{x}$ and $u_{z}$ are two distinct nonzero codewords from $C^{\prime}$, both of Hamming weight at least $d^{\prime}$ and at Hamming distance at least $d^{\prime}$ apart. Therefore, their bitwise OR is at least $\left\lceil\frac{3 d^{\prime}}{2}\right\rceil$.

As an illustration of the previous theorem, we present below a recordbreaking code. We also provide a table of a few quantum codes which, although they just equal previously known codes in terms of parameters, present the advantage of being obtained through binary codes. The generator matrices of the corresponding $C^{\prime \prime}$ s can be found in the appendix. 
Table 1: Some quantum codes obtained by theorems 1, 2

\begin{tabular}{|r|l|l|l|l|l|l|c|}
\hline$n$ & $k$ & $k^{\prime}$ & $d$ & $d^{\prime}$ & $K$ & $d_{[[n, k]]}$ & Remarks \\
\hline 8 & 4 & 7 & 4 & 2 & 3 & 3 & \\
12 & 6 & 10 & 4 & 2 & 4 & 3 & \\
12 & 6 & 11 & 4 & 2 & 5 & 3 & \\
14 & 7 & 9 & 4 & 2 & 2 & 4 & $d_{2}^{\prime}>\left\lceil\frac{3 d^{\prime}}{2^{\prime}}\right\rceil$, apply Th 2 \\
14 & 7 & 10 & 4 & 2 & 3 & 4 & $d_{2}^{\prime}>\left\lceil\frac{3 d^{\prime}}{2}\right\rceil$, apply Th 2 \\
18 & 9 & 12 & 6 & 4 & 3 & 6 & optimal \\
\hline
\end{tabular}

Example 1 We extend the QR $[18,9,6]$-code $C$ to a $C^{\prime}[18,12,4]$ with generator matrix written below. Applying Steane's construction we obtain a $[[18,3,6]]$-code. The highest achievable minimal distance $d$ of a $[[18,3, d]]$ code is 5 or 6 [3]. This shows that $d=6$, thus closing the gap on $d$.

111111000000000000
001011100000101000
001001011000110000
011100000100110000
011010000000011100
011001001010001000
001000001100101010
001000001100010101
011000000111000100
110000101000000000
000000001000110001
000000010100001001

In Table $1, K$ and $d_{[[n, k]]}$ are the dimension and the minimum distance of a quantum code obtained by theorems [1, 2 . 


\section{An improvement}

We need the following notion introduced by Wei [12]. The $i$-th generalized distance $d_{i}$ of a linear code $C[n, k]$ is the minimum size of the support of a i-th dimensional subcode of $C$. For the best known bounds on generalized weights consult [5, 6, 11, 12].

Clearly, for $i=1, d_{1}$ is the minimum distance of the code; for $i=2, d_{2}$ is the minimum weight of the bitwise OR of two different nonzero codewords. It follows easily from the Griesmer bound (see e.g. [7, Chap.17]), that $d_{2} \geq$ $\lceil 3 d / 2\rceil$. We are ready now for the improvement.

Theorem 2 With the notation of the previous theorem, $\mathcal{G}$ generates a quantum code of parameters $\left[\left[n, k+k^{\prime}-n, \min \left(d, d_{2}^{\prime}\right)\right]\right]$, where $d_{2}^{\prime}$ is the second generalized distance of $C^{\prime}$.

Proof Recalling the proof of the previous theorem, the only thing left to be shown is that if rows of $\left(G^{\prime} \mid P G^{\prime}\right)$ are involved in the generation of $\mathbf{u}$, then the generalized weight of $\mathbf{u}$ is $\geq d_{2}^{\prime}$. In this case both $u_{x}$ and $u_{z}$ are in $C^{\prime}$, and distinct since $P$ has no fix point, and the assertion follows from the definition of the generalized distance. This bound is at least as strong as the previous one, since $d_{2}^{\prime} \geq 3 d^{\prime} / 2$ by the remark preceding the theorem.

\section{Some new quantum codes}

Steane [10] proved that the primitive $\mathrm{BCH}$ codes of length $2^{m}-1$ contain their duals if and only if their designed distance $d=2 t+1$ satisfies $d \leq 2^{\lceil m / 2\rceil}-1$. It follows from [7, Corollary 8, Chapter 9] that in this case the codes have parameters $\left[2^{m}-1,2^{m}-1-m t, 2 t+1\right]$. Moreover, these codes are nested, i.e. form a chain for the inclusion relation when $t$ increases. Extending them with a parity bit, we derive the following families using Theorem $\mathbb{1}$ :

$$
\begin{array}{rcl}
F_{0}\left(d=6 \ell, d^{\prime}=4 \ell\right) & {\left[\left[2^{m}, 2^{m}-(5 \ell-2) m-2,6 \ell\right]\right]} & \text { for } 6 \ell \leq 2^{\lceil m / 2\rceil} ; \\
F_{2}\left(d=6 \ell+2, d^{\prime}=4 \ell+2\right) & {\left[\left[2^{m}, 2^{m}-5 \ell m-2,6 \ell+2\right]\right]} & \text { for } 6 \ell+2 \leq 2^{\lceil m / 2\rceil} ; \\
F_{3}\left(d=6 \ell+4, d^{\prime}=4 \ell+2\right) & {\left[\left[2^{m}, 2^{m}-(5 \ell+1) m-2,6 \ell+3\right]\right]} & \text { for } 6 \ell+4 \leq 2^{\lceil m / 2\rceil} .
\end{array}
$$


Theorem 6 of [3] shows how to construct an [[n,K+1,d-1]] code from an $[[n, K, d]]$ code. Using it we construct from $F_{0}$ the following family:

$$
F_{5} \quad\left[\left[2^{m}, 2^{m}-(5 \ell+3) m-1,6 \ell+5\right]\right] \text { for } 6 \ell+6 \leq 2^{\lceil m / 2\rceil} .
$$

It is tempting to conjecture the existence of families of codes with parameters

$$
F_{a}=\left[\left[2^{m}, 2^{m}-(5 \ell+a-2) m+b, 6 \ell+a\right]\right],
$$

where $a=0,1,2,3,4,5$ and $b$ is a small integer constant. Using Theorem 2 we construct such a family for $a=4$. The case $a=1$ remains open.

Theorem 3 For $6 \ell+4 \leq 2^{\lceil m / 2\rceil}$ there exist quantum codes with parameters

$$
F_{4}=\left[\left[2^{m}, 2^{m}-(5 \ell+2) m-1,6 \ell+4\right]\right] .
$$

Proof Consider the extended BCH code, $C\left[2^{m}, 2^{m}-(3 \ell+1) m-1,6 \ell+4\right]$. As the code $C^{\prime}$ we take the union of the code $C_{1}=\left[2^{m}, 2^{m}-(2 \ell+1) m-1,4 \ell+4\right]$ with any coset of $C_{1}$, say $C_{2}$, in the code $\left[2^{m}, 2^{m}-2 \ell m-1,4 \ell+2\right]$. This union is a $C^{\prime}=\left[2^{m}, 2^{m}-2(\ell+1) m, 4 \ell+2\right]$ code. However, $d_{2}^{\prime} \geq 6 \ell+4>$ $3 d^{\prime} / 2=6 \ell+3$. Indeed, consider the bitwise OR of any two codewords of $C^{\prime}$. If they both have weight $4 \ell+2$ they belong to $C_{2}$, hence are at least at distance $4 \ell+4$ apart, yielding a generalized weight at least $6 \ell+4$. Otherwise, one of the words has weight at least $4 \ell+4$, and even if the second one is of weight $4 \ell+2$, since their distance is at least $4 \ell+2$, this again guarantees a minimum generalized weight of at least $6 \ell+4$.

\section{Asymptotical behaviour}

Let us consider now the asymptotical-in $n$ - non constructive behavior of codes obtained by use of Theorem 2. Let $R_{Q}(\mathcal{C})=K / n$ and $\delta_{Q}(\mathcal{C})=d / n$ stand for the rate and relative minimum distance of $\mathcal{C}=[[n, K, d]]$. We are interested in

$$
R_{Q}\left(\delta_{Q}\right)=\limsup _{n \rightarrow \infty} R_{Q}(\mathcal{C})
$$

where the limit is taken over all codes with $\delta_{Q}(\mathcal{C}) \geq \delta_{Q}$.

The best known lower bound on $R_{Q}$ is obtained [3] via codes over $G F(4)$ :

$$
R_{Q} \geq 1-\delta_{Q} \log _{2} 3-H\left(\delta_{Q}\right)
$$


where $H(x)=-x \log _{2} x-(1-x) \log _{2}(1-x)$ is the binary entropy function. For the best upper bounds see [1].

For binary constructions, Calderbank and Shor [4] proved a weaker bound

$$
R_{Q}^{b} \geq 1-2 H\left(\delta_{Q}\right)
$$

It is fairly easy to show that Theorem 1 yields

$$
R_{Q}^{b} \geq 1-H\left(\delta_{Q}\right)-H\left(2 \delta_{Q} / 3\right),
$$

which is better than (2).

Theorem 4

$$
R_{Q}^{b} \geq 1-\frac{\delta_{Q} \log _{2} 3}{2}-3 \frac{H\left(\delta_{Q}\right)}{2} .
$$

Proof It follows along the lines of the standard proofs, see e.g. [1, Section 5]. Let $k=\lfloor R n\rfloor, k^{\prime}=\left\lfloor R^{\prime} n\right\rfloor, d=d_{2}^{\prime}=\left\lfloor\delta_{Q} n\right\rfloor$ be the parameters of $C$ and $C^{\prime}$. For given $n, k$ and $k^{\prime}$, consider two families of codes:

$$
\begin{gathered}
\mathcal{C}^{(1)}=\left\{C[n, k] \text { such that } C^{\perp} \subseteq C\right\} \\
\mathcal{C}^{(2)}=\left\{C^{\prime}\left[n, k^{\prime}\right] \text { such that } C \subset C^{\prime} \text { for at least one } C \in \mathcal{C}^{(1)}\right\} .
\end{gathered}
$$

The proof consists of three steps. We first prove that almost all codes in $\mathcal{C}^{(1)}$ lie above the Varshamov Gilbert (VG) bound (see e.g. [7]); then that almost all codes in $\mathcal{C}^{(2)}$ lie above an analog of the VG bound for the second generalized distance; finally we combine these two results to construct binary quantum codes satisfying the theorem. Arguments from [四] yield that

a) every non-zero vector of even weight belongs to the same number, say A, of codes from $\mathcal{C}^{(1)}$;

b) every pair of non-zero vectors of even weight belongs to the same number, say $\mathrm{B}$, of codes from $\mathcal{C}^{(2)}$;

c) every code in $\mathcal{C}^{(1)}$ belongs to the same number of codes in $\mathcal{C}^{(2)}$.

By hypothesis a),

$$
\left(2^{n-1}-1\right) A=\left(2^{k}-1\right)\left|\mathcal{C}^{(1)}\right|
$$

and if

$$
A \sum_{j=1}^{(d-1) / 2}\left(\begin{array}{c}
n \\
2 j
\end{array}\right) \leq A \frac{\left(2^{n-1}-1\right)}{n\left(2^{k}-1\right)}=\left|\mathcal{C}^{(1)}\right| / n
$$


then at least $(1-1 / n)\left|\mathcal{C}^{(1)}\right|$ codes from $\mathcal{C}^{(1)}$ satisfy the VG bound

$$
R \geq 1-H\left(\delta_{Q}\right)
$$

Consider now the pairs of non-zero even-weight vectors with bitwise OR equal exactly to some vector of weight $t$. Their number is less than

$$
\frac{1}{2} \sum_{j=0}^{[t / 2]}\left(\begin{array}{c}
t \\
2 j
\end{array}\right) 2^{2 j-1}=\frac{1}{8}\left((1+2)^{t}+(1-2)^{t}\right) \leq \frac{1}{8}\left(3^{t}+1\right) .
$$

The total number of such pairs for $t \leq d$ is at most

$$
\frac{1}{8} \sum_{t=1}^{d}\left(\begin{array}{l}
n \\
t
\end{array}\right)\left(3^{t}+1\right)
$$

By argument b)

$$
\left(\begin{array}{c}
2^{n-1}-1 \\
2
\end{array}\right) B=\left(\begin{array}{c}
2^{k^{\prime}}-1 \\
2
\end{array}\right)\left|\mathcal{C}^{(2)}\right|
$$

and analogously to the previous argument we have that at least $(1-1 / n)\left|\mathcal{C}^{(2)}\right|$ codes from $\mathcal{C}^{(2)}$ satisfy

$$
R^{\prime} \geq 1-\frac{\delta_{Q} \log _{2} 3}{2}-\frac{H\left(\delta_{Q}\right)}{2}
$$

Finally, argument c) implies the existence of a code satisfying both (任) and (5). By Theorem 2 , this yields a binary quantum code $\left[\left[n,\left(R+R^{\prime}-1\right) n, \delta_{Q} n\right]\right]$ as stated in Theorem 4.

\section{References}

[1] A.Ashikhmin and S.Litsyn, "Upper bounds on the size of quantum codes," IEEE Trans. Info. Theory, to appear.

[2] A.R. Calderbank, E.M. Rains, N.J.A. Sloane and P.W. Shor, "Quantum error correction and orthogonal geometry", Phys. Rev. Lett., vol.78, pp. 405-409, 1997. 
[3] A.R. Calderbank, E.M. Rains, N.J.A. Sloane and P.W. Shor, "Quantum error correction via codes over GF(4)," IEEE Trans. Info. Theory, vol.44, pp.1369-1387, 1998.

[4] A.R. Calderbank and P.W. Shor, "Good quantum error-correcting codes exist", Phys. Rev. A, vol.54, pp.1098-1105, 1996.

[5] G.Cohen, S.Litsyn and G.Zémor, "Upper bounds on generalized distances," IEEE Trans. Info. Theory, vol.40, pp.2090-2092, 1994.

[6] T.Helleseth, T.Kløve, V.I.Levenshtein, and Ø.Ytrehus, "Bounds on the minimum support weights," IEEE Trans. Info. Theory, vol.41, pp.432440, 1995.

[7] F.J.MacWilliams and N.J.A.Sloane, The theory of error-correcting codes, Elsevier, 1977.

[8] A.M. Steane, "Multiple particle interference and quantum error correction," Proc. Roy. Soc. Lond. A, vol. 452, pp. 2551-2577, 1996.

[9] A.M. Steane, "Simple quantum error correcting codes", Phys. Rev. Lett., vol. 77, pp. 793-797, 1996.

[10] A.M. Steane, "Enlargement of Calderbank-Shor-Steane quantum codes," quantum-ph/9802061, 24 Feb 1998.

[11] M.A.Tsfasman and S.G.Vladuts, "Geometric approach to higher weights," IEEE Trans. Info. Theory, vol.41, pp.1564-1588, 1995.

[12] V.Wei, "Generalized Hamming weights for linear codes," IEEE Trans. Info. Theory, vol.37, pp.1412-1418, 1991. 


\section{Appendix}

Generator matrices for codes $C^{\prime}$ in Table 1 .

$C^{\prime}[12,10,2]$ (two possibilities)

$\left(\begin{array}{l}111100000000 \\ 001111000000 \\ 000011110000 \\ 000000111100 \\ 000000001111 \\ 010101010101 \\ 110000000000 \\ 101000000000 \\ 100000001000 \\ 100000000001\end{array}\right)$,

$\left(\begin{array}{l}111100000000 \\ 001111000000 \\ 000011110000 \\ 000000111100 \\ 000000001111 \\ 010101010101 \\ 000000001010 \\ 001000010010 \\ 001010000100 \\ 110000000000\end{array}\right)$, 
$C^{\prime}[14,9,2]$

$\left(\begin{array}{l}11110000000000 \\ 00111100000000 \\ 10101010000000 \\ 00000001111000 \\ 00000000011110 \\ 00000001010101 \\ 1111111111111 \\ 10001000000001 \\ 01000000100001\end{array}\right)$,

$C^{\prime}[14,10,2]$

$\left(\begin{array}{l}11110000000000 \\ 00111100000000 \\ 10101010000000 \\ 00000001111000 \\ 00000000011110 \\ 00000001010101 \\ 1111111111111 \\ 10001000000001 \\ 01000000100001 \\ 10100000000000\end{array}\right)$,

